\section{AN AUDIT OF CATHETER-ASSOCIATED BLOODSTREAM INFECTIONS IN A TERTIARY PAEDIATRIC INTENSIVE CARE UNIT}

M. McGuigan, R. Guhadasan, D. Das, S. Paulus, A. Darbyshire

PICU, Alder Hey Childrens Hospital, Liverpool, UK

Background: We studied the incidence of catheterassociated bloodstream infections (CABSI) in a tertiary specialist 23-bedded paediatric intensivecare unit (PICU). Ongoing surveillance of CABSI incidence is important for continuous quality improvement and maintaining patient safety. There is little published data on the incidence of CABSI in UK paediatric intensive care units.

Methods: We prospectively collected daily clinical and laboratory data on all PICU admissions for four months, retrospectively using electronic patient records to supplement missing data. All positive blood cultures were identified and the total number of catheter days was calculated. We used the UK National Patient Safety Association "Matching Michigan" (MM) case definitions to identify bloodstream infections and CABSI and determined the rate of CABSI.

Results: There were 429 PICU admissions, giving a total of 1578 catheter days. There were 39 positive blood cultures from all sources. The incidence of CABSI was 6.33 per 1000 catheter days. The average length of stay for all patients and those with CABSI was 5 and 17 days respectively.

Conclusion: Although this was a time-limited preliminary audit, our calculated CABSI incidence rate was comparable to that described in the literature. We report some difficulty in using MM case definitions to evaluate our data set and discuss the utility of Systemic Inflammatory Response Syndrome criteria in our population, particularly in post-operative cardiac patients. This audit forms an important first step in the implementation of "care bundles" to reduce modifiable risk factors. It is imperative to establish effective ongoing electronic surveillance of Health Care Associated Infections.

\section{WHAT IS THE MOST APPROPRIATE ANTIBIOTIC TO TREAT LATE ONSET NEONATAL INFECTIONS?}

\author{
S. Jyothi, I. Lakshminarayna
}

\section{Paediatrics, University Hospital of North Staffordshire, Stoke, UK}

Introduction: Late onset neonatal infection (systemic infection after 48 hours) is an important cause of morbidity and mortality in neonates. Coagulase negative staphylococci (CoNS) is responsible for more than half of these infections but there is no consensus on the most effective antibiotics.

Aim: To evaluate the efficacy and suitability of our antibiotic guideline to treat late onset neonatal sepsis and to compare our guidelines with other level 3 neonatal units in UK.

Methods: A list of all positive blood culture results were obtained from microbiology department over one year period (June 2008-June 2009). The notes of these babies were reviewed. We also collected data on the antibiotics used in other tertiary NICU in the UK for late onset sepsis.

Results: A total of 22 babies were identified with positive blood cultures during their stay in NICU over 1 year period. 18 of these $(81 \%)$ had grown CONS and 4 grew other organisms including Group B Streptoccci, enterococci and staphylococcus aureus. Of those that grew CoNS, all were sensitive to Vanocomycin. $13 / 18 \quad(72 \%)$ were resistant to flucloxacillin and $10(55 \%)$ were resistant to Gentamycin.

Conclusion: Our study indicates that the most commonest organism isolated is CoNS. There is widespread resistance to flucloxacillin and gentamicin. Vancomycin is sensistive in all cases but its use is reserved due to concerns regarding development of resistance. The antibiotics used will depend on the prevalent micro organisms in the area and their resistance. Neonatalologists will need to work with Microbiologists to develop local guidelines. 